\title{
Fadiga por compaixão em profissionais de saúde durante a pandemia da Covid-19:
}

\section{Revisão integrativa}

\author{
Compassion fatigue in healthcare professionals during the Covid-19 pandemic: Integrative review \\ Fatiga por compasión en los profesionales de la salud durante la pandemia de Covid-19: Revisión
}

integradora

Recebido: 10/06/2021 | Revisado: 17/06/2021 | Aceito: 18/06/2021 | Publicado: 30/06/2021

\author{
Marli Aparecida Reis Coimbra \\ ORCID: https://orcid.org/0000-0001-6230-9351 \\ Universidade Federal do Triângulo Mineiro, Brasil \\ E-mail: marli.apr.coimbra@gmail.com \\ Érica Midori Ikegami \\ ORCID: https://orcid.org/0000-0002-7262-5881 \\ Universidade Federal do Triângulo Mineiro, Brasil \\ E-mail: erica.ikegami@gmail.com \\ Ana Paula de Freitas Fernandes \\ ORCID: https://orcid.org/0000-0003-2280-4125 \\ Universidade Federal do Triângulo Mineiro, Brasil \\ E-mail: paulafreitasfernandes1@gmail.com \\ Jair Sindra Virtuoso Júnior \\ ORCID: https://orcid.org/0000-0001-7602-1789 \\ Universidade Federal do Triângulo Mineiro, Brasil \\ E-mail: jair.junior@uftm.edu.br \\ Lúcia Aparecida Ferreira \\ ORCID: https://orcid.org/0000-0001-6469-5444 \\ Universidade Federal do Triângulo Mineiro, Brasil \\ E-mail: lap2ferreira@yahoo.com.br
}

\begin{abstract}
Resumo
Introdução: Os profissionais de saúde atendem todos os tipos de emergência e pacientes, como os acometidos pela COVID-19, enfrentando situações críticas e estressantes que podem favorecer o desenvolvimento de fadiga por compaixão (FC). Objetivo: Caracterizar a produção científica e sintetizar os aspectos mais relevantes sobre FC em profissionais da saúde durante a pandemia da COVID-19. Método: Revisão integrativa da literatura nas bases de dados BVS, MEDLINE via PubMed, PsycINFO, CINAHL, Web of Science e Scopus. Para a estratégia de busca foram utilizados os descritores: "compassion fatigue", "health personnel" e "coronavirus infections". Considerou-se como critérios de inclusão os artigos originais, publicados de março de 2020 a fevereiro de 2021, disponíveis eletronicamente na íntegra, sem restrição de idioma e que avaliaram a FC em profissionais de saúde durante a pandemia da COVID-19. Resultados: Foram elegíveis 13 artigos e, da análise destes, emergiram duas categorias: presença de FC e fatores relacionados à FC. Conclusão: Profissionais de saúde apresentaram FC, com predominância em mulheres, médicos, enfermeiros e aqueles atuantes em unidades específicas de atendimento a pacientes com COVID-19. Entre os fatores relacionados à FC estavam questões de saúde mental, éticas, ocupacionais e sociodemográficas. Os achados indicam a necessidade de avaliação, monitoramento e tratamento dessa condição nos profissionais de saúde durante a pandemia.
\end{abstract}

Palavras-chave: Fadiga por compaixão; Estresse psicológico; Infecções por coronavirus; Pessoal de saúde; Saúde mental.

\begin{abstract}
Introduction: Health professionals attend to all types of emergencies and patients, such as those affected by COVID19, facing critical and stressful situations that can favor the development of compassion fatigue (CF). Objective: To characterize the scientific production and synthesize the most relevant aspects of CF in health professionals during the COVID-19 pandemic. Methods: Integrative literature review in the databases BVS, MEDLINE via PubMed, PsycINFO, CINAHL, Web of Science and Scopus. For the search strategy, the following descriptors were used: "compassion fatigue", "health personnel" and "coronavirus infections". The inclusion criteria were original articles published from March 2020 to February 2021, available electronically in their entirety, without language restrictions, and that assessed CF in health professionals during the COVID-19 pandemic. Results: Thirteen articles were eligible and, from the analysis of these, two categories emerged: presence of CF and factors related to CF. Conclusion: Health professionals presented $\mathrm{CF}$, predominantly in women, physicians, nurses and those working in specific care units for patients with COVID-19. Among the factors related to CF were mental health, ethical, occupational and
\end{abstract}


sociodemographic issues. The findings indicate the need for assessment, monitoring and treatment of this condition in health professionals during the pandemic.

Keywords: Compassion fatigue; Stress psychological; Coronavirus infections; Health personnel; Mental health.

\section{Resumen}

Introducción: Los profesionales de la salud atienden todo tipo de emergencias y los pacientes, como los afectados por COVID-19, enfrentan situaciones críticas y estresantes que pueden favorecer el desarrollo de la fatiga compasiva (FC). Objetivo: Caracterizar la producción científica y sintetizar los aspectos más relevantes de la FC en los profesionales de la salud durante la pandemia de COVID-19. Métodos: Revisión integrativa de la literatura en las bases de datos BVS, MEDLINE vía PubMed, PsycINFO, CINAHL, Web of Science y Scopus. Para la estrategia de búsqueda se utilizaron los siguientes descriptores: "compassion fatigue", "health personnel" e "coronavirus infections". Los criterios de inclusión fueron artículos originales publicados desde marzo de 2020 hasta febrero de 2021, disponibles electrónicamente en su totalidad, sin restricciones de idioma, y que evaluaran FC en profesionales de la salud durante la pandemia de COVID-19. Resultados: Fueron elegibles trece artículos y del análisis de los mismos surgieron dos categorías: presencia de FC y factores relacionados con FC. Conclusión: Los profesionales de la salud presentaron FC, predominantemente en mujeres, médicos, enfermeras y quienes laboran en unidades de atención específicas para pacientes con COVID-19. Entre los factores relacionados con la FC se encontraban cuestiones de salud mental, éticas, ocupacionales y sociodemográficas. Los hallazgos indican la necesidad de evaluación, seguimiento y tratamiento de esta condición en los profesionales de la salud durante la pandemia.

Palabras clave: Desgaste por empatía; Estrés psicológico; Infecciones por coronavirus; Personal de salud; Salud mental.

\section{Introdução}

Desde que foi declarada como pandemia, a COVID-19 causada pelo vírus SARS-CoV-2 tem afetado o mundo todo em diferentes proporções (Committee for the Coordination of Statistical Activities, 2020), devido ao seu alto potencial de propagação e presença de fatores de risco que aumentam a suscetibilidade à infecção, que varia de casos assintomáticos a quadros de pneumonia e morte (World Health Organization, 2020). Até o dia 11 de maio de 2021, a Organização Mundial da Saúde tinha recebido a notificação de 158.651.638 casos confirmados de COVID-19, incluindo 3.299.764 mortes no mundo (World Health Organization, 2021).

Medidas de saúde como a lavagem das mãos, a etiqueta respiratória ao tossir e espirrar, o uso de máscaras e o distanciamento social têm sido orientadas a toda população com objetivo de conter a propagação do vírus (World Health Organization, 2020). Entretanto, devido à necessidade de prestar assistência a casos suspeitos ou diagnosticados de COVID-19, os profissionais de saúde nos diversos cenários clínicos não puderam seguir as recomendações de permanecer em casa, ficando expostos à doença (Teixeira et al., 2020).

Além do risco de contágio, os trabalhadores da saúde na pandemia foram desafiados por condições críticas (Vagni et al., 2020b) como a escassez de equipamentos de proteção individual (Ruiz-Fernández et al., 2020), jornadas de trabalho extensas, abordagem de quadros graves em meio às incertezas científicas e contato constante com o sofrimento e a morte dos pacientes (Vagni et al., 2020b). Estudo de revisão sistemática identificou que esses profissionais apresentaram ansiedade, depressão e estresse (Vizheh et al., 2020), indicando que a exposição constante a situações de perturbação causa riscos à saúde mental (Teixeira et al., 2020).

As mudanças onerosas nas respostas fornecidas pelas equipes médicas na crise de doenças infecciosas emergentes (Vizheh et al., 2020), também podem favorecer o surgimento da fadiga por compaixão (FC). Trata-se do aspecto negativo da qualidade de vida profissional, que envolve características do esgotamento, como exaustão, sentimentos de insatisfação, raiva e depressão; e do estresse traumático secundário, que incluem temores e traumas relacionados à prestação do cuidado (Stamm, 2010).

A FC também pode ser denominada de Estresse Traumático Secundário (ETS) (Figley, 2002; Stamm, 2010) ou Traumatização Vicária (TV) (McCann \& Pearlman, 1990; Stamm, 2010), sendo os três termos correlatos e aceitos, devido à ausência de limites que demarquem suas diferenças (Stamm, 2010). O desenvolvimento dessa condição é caracterizado pela 
empatia e compaixão, componentes essenciais do cuidado humano, mas que podem trazer repercussões negativas aos profissionais, a partir do momento que vivenciar lembranças traumáticas e suportar o sofrimento do outro se torna excessivo (Figley, 2002).

A literatura mostra que a FC tem sido amplamente investigada em enfermeiros (Cavanagh et al., 2020) e associada a alguns fatores como o sexo feminino (Aslan et al., 2021; Borges et al., 2019), menor idade e tempo de experiência na profissão (Borges et al., 2019) estresse relacionado à ocupação e condições de trabalho (Aslan et al., 2021). Os estudos enfatizam a importância dessa condição na saúde mental e ocupacional dos profissionais de saúde (Aslan et al., 2021; Borges et al., 2019; Cavanagh et al., 2020; Vizheh et al., 2020), independentemente dos fatores associados e da não inclusão da FC na Classificação Internacional de Doenças (CID - 10) ou no Manual Diagnóstico e Estatístico de Transtornos Mentais (DSM-V).

A relevância social deste estudo pauta-se na incapacidade laboral e mental que a FC pode causar nos profissionais de saúde, expostos à pandemia da COVID-19. A ampliação e sumarização dos conhecimentos sobre os danos relacionados à FC poderá contribuir para ressignificar as ações e estratégias, com objetivo de prevenir e/ou reduzir essa condição. Diante do exposto, o objetivo do estudo foi caracterizar a produção científica e sintetizar os aspectos mais relevantes sobre FC em profissionais da saúde durante a pandemia da COVID-19.

\section{Metodologia}

Trata-se de uma revisão integrativa da literatura que foi conduzida em seis etapas: I) identificação do tema e elaboração da pergunta norteadora; II) busca dos artigos na literatura científica e estabelecimento dos critérios de inclusão e exclusão dos estudos; III) coleta de dados; IV) análise crítica dos artigos incluídos na revisão; V) análise e interpretação dos resultados e VI) apresentação da revisão (Mendes et al., 2008).

Para orientar a revisão integrativa, foi formulada a seguinte questão: "Quais as características da produção científica e os aspectos mais relevantes sobre FC em profissionais de saúde durante a pandemia da COVID-19?”. Foram incluídos artigos originais, publicados de março de 2020 a fevereiro de 2021, disponíveis eletronicamente na íntegra, sem restrição de idioma, que investigaram a FC em profissionais de saúde durante a pandemia da COVID-19. Foram excluídos os artigos duplicados, editoriais, artigos de opinião e de revisão, cartas, comentários, notas, teses, dissertações, manuais e trabalhos publicados em anais de eventos científicos.

A coleta de dados foi realizada em março de 2021 em seis bases de dados: Biblioteca Virtual em Saúde (BVS), Medical Literature Analysis and Retrievel System Online (MEDLINE) via PubMed, PsycINFO, Cumulative Index to Nursing and Allied Health Literature (CINAHL), Web of Science e Scopus. Para elaborar a estratégia de busca, foi realizada a consulta nos Descritores em Ciências da Saúde (DeCS), sendo selecionados os descritores: "health personnel", "coronavirus infections" e "compassion fatigue". Após a busca no DeCS foram consultados os descritores no Medical Subject Headings (MESH), PsycINFO Thesaurus e CINAHL Headings.

Foram realizadas as combinações dos descritores e seus sinônimos correspondentes utilizando o operador booleano OR e construída a busca final com AND, de acordo com cada base, da seguinte maneira:

1. health personnel OR personnel, health OR health care providers OR health care provider OR provider, health care OR healthcare providers OR healthcare provider OR provider, healthcare OR healthcare workers OR healthcare worker OR health care professionals OR health care professional OR professional, health care;

2. coronavirus OR coronavirus infections OR COVID-19 OR SARS-CoV-2 OR coronaviruses OR coronavirus infection OR infection, coronavirus OR infections, coronavirus;

3. compassion fatigue $\mathrm{OR}$ fatigue, compassion $\mathrm{OR}$ vicarious trauma $\mathrm{OR}$ trauma, vicarious $\mathrm{OR}$ traumas, vicarious $\mathrm{OR}$ vicarious traumas OR secondary trauma OR secondary traumas OR trauma, secondary OR traumas, secondary OR secondary 
traumatization OR secondary traumatizations OR traumatization, secondary OR traumatizations, secondary OR secondary traumatic stress OR stress, secondary traumatic OR stresses, secondary traumatic OR traumatic stress, secondary OR vicarious traumatization OR traumatization, vicarious.

\section{1 AND 2 AND 3.}

Após as buscas, dois pesquisadores de forma independente realizaram a leitura dos títulos e resumos e, posteriormente, dos artigos na íntegra considerando os critérios de seleção (Mendes et al., 2008). Os dados extraídos e considerados para análise crítica foram os itens propostos em instrumento utilizado em revisões integrativas: título, autores, ano e país de publicação, objetivo, características metodológicas (tipo de publicação, amostra, instrumentos de avaliação) e principais resultados (Ursi, 2005). Este último item foi agrupado em categorias de acordo com os aspectos mais relevantes identificados nos artigos. O instrumento mencionado também orientou a avaliação do rigor metodológico (Ursi, 2005). Todos os estudos elegíveis foram avaliados quanto à classificação do nível de evidência (Stetler et al., 1998).

\section{Resultados}

Foram encontrados 172 estudos, dos quais 82 eram duplicados. Após a leitura de títulos e resumos, 72 foram excluídos, restando 18 artigos para a leitura na íntegra. Após esta última etapa, 13 artigos foram considerados elegíveis para análise na revisão. A Figura 1 mostra o diagrama das etapas de busca e seleção dos estudos de acordo com as recomendações do Preferred Reporting Items for Systematic Reviews and Meta-Analyses (PRISMA) (Page et al., 2021).

Figura 1 - Diagrama baseado no PRISMA referente às etapas de busca e seleção dos estudos.

\section{Identificação de estudos por meio de bancos de dados}

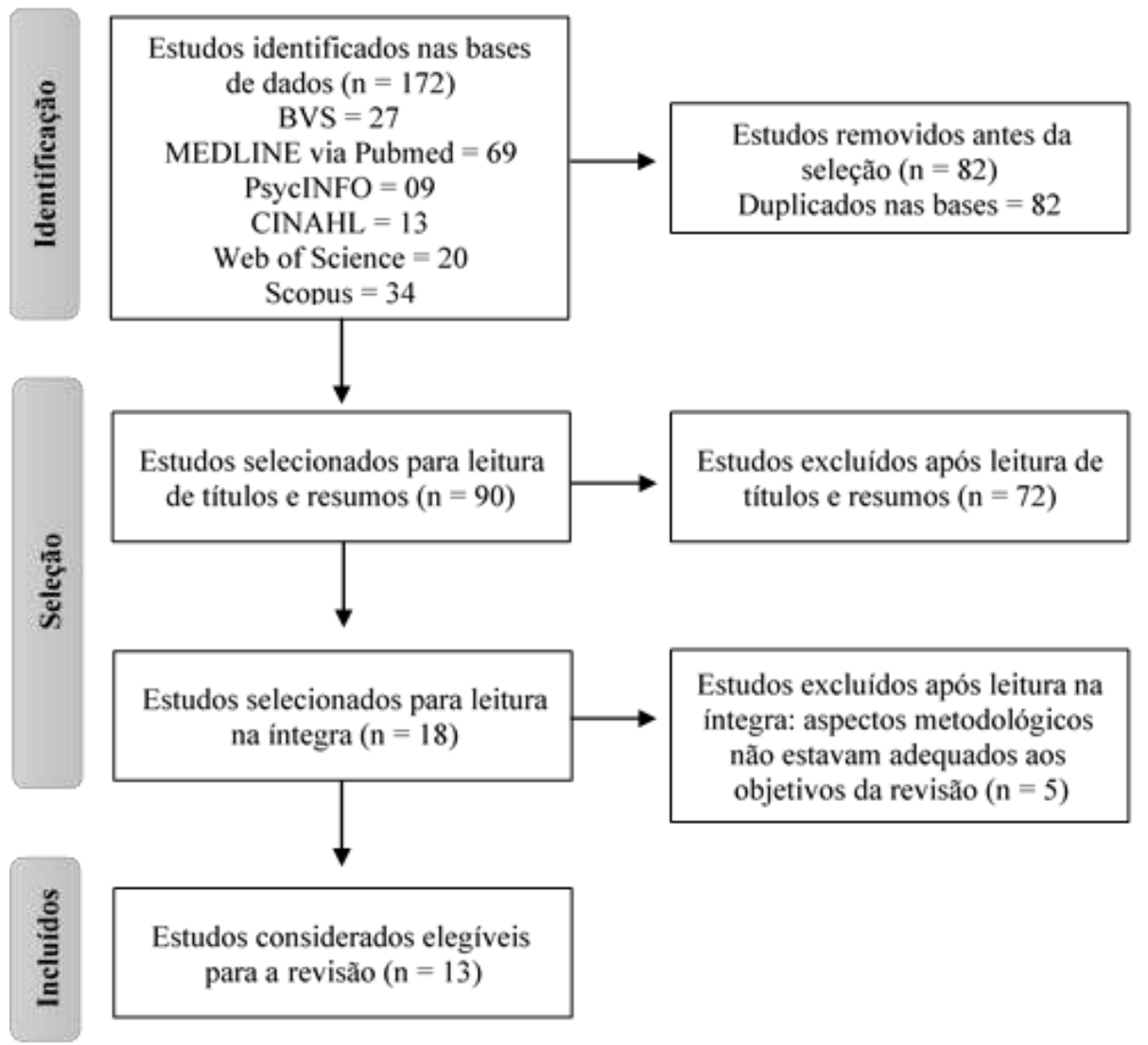

Fonte: Dados da pesquisa (2021). 
A síntese dos artigos mostrou que todos os estudos identificados eram transversais (nível IV de evidência científica) (Stetler et al., 1998), publicados em inglês (92,3\%) e espanhol (7,7\%), no ano de 2020 (76,9\%) e 2021 (23,1\%), com predomínio de pesquisas realizadas em países europeus, como a Itália (Quadro 1).

Quanto as características da amostra, verificou-se que todos os estudos tiveram predomínio de mulheres, enfermeiros e médicos. Dos 13 estudos, nove (69,2\%) utilizaram o instrumento Professional Quality of Life Scale (ProQOL) que avalia dois aspectos: um negativo, a FC, dividida em ETS e burnout, e um positivo, a satisfação por compaixão. O segundo questionário adotado nos outros quatro estudos (30,8\%) foi o Secondary Traumatic Stress Scale (STSS) que investiga os fatores de intrusão, evitação e excitação, ou somente intrusão e excitação (Quadro 1).

Quadro 1 - Caracterização da produção científica sobre FC em profissionais de saúde durante a pandemia da COVID-19.

\begin{tabular}{|c|c|c|}
\hline Título, autores, ano e país do estudo & Objetivo & $\begin{array}{l}\text { Características metodológicas (tipo de } \\
\text { estudo, amostra, instrumento de FC) }\end{array}$ \\
\hline $\begin{array}{l}\text { Professional quality of life and mental health } \\
\text { outcomes among health care workers exposed } \\
\text { to Sars-Cov-2 (Covid-19) (Buselli et al., 2020) } \\
\text { - Itália. }\end{array}$ & $\begin{array}{l}\text { Identificar a contribuição de variáveis } \\
\text { contextuais, pessoais e de trabalho entre } \\
\text { trabalhadores de linha de frente e de segunda } \\
\text { linha nas dimensões da vida profissional e } \\
\text { analisar o possível impacto dessas dimensões } \\
\text { na saúde mental dos profissionais de saúde. }\end{array}$ & $\begin{array}{l}\text { Transversal, } \mathrm{n}=265 \text { (médicos, enfermeiros e } \\
\text { auxiliares de saúde), } \mathrm{H}=31,7 \% \text { e } \mathrm{M}=68,9 \% \text { com } \\
40,4 \pm 11,2 \text { anos. Foi utilizado o instrumento } \\
\text { ProQOL. }\end{array}$ \\
\hline $\begin{array}{l}\text { Psychological symptoms in health } \\
\text { professionals in Spain after the first wave of the } \\
\text { COVID-19 Pandemic (Dosil et al., 2020) - } \\
\text { Espanha. }\end{array}$ & $\begin{array}{l}\text { Avaliar os níveis de estresse, ansiedade, } \\
\text { depressão, estresse pós-traumático e FC em } \\
\text { profissionais de saúde. }\end{array}$ & $\begin{array}{l}\text { Transversal, } \mathrm{n}=973 \text { (médicos, enfermeiros, } \\
\text { auxiliares/técnicos), } \mathrm{H}=16,5 \%, \mathrm{M}=82,9 \% \text { e uma } \\
\text { pessoa considerada não binária, com idades } \\
\text { entre 18-25, 26-35, 36-55 e maiores de } 56 \text { anos. } \\
\text { Foi utilizado o instrumento ProQOL. }\end{array}$ \\
\hline $\begin{array}{l}\text { Moral injury in health-care workers during } \\
\text { COVID-19 pandemic (Litam \& Balkin, 2020) - } \\
\text { Estados Unidos da América. }\end{array}$ & $\begin{array}{l}\text { Examinar até que ponto o dano moral afetou os } \\
\text { profissionais de saúde durante a COVID-19. }\end{array}$ & $\begin{array}{l}\text { Transversal, } \mathrm{n}=109 \text { (médicos, enfermeiros e } \\
\text { outros profissionais), } \mathrm{H}=24 \% \text { e } \mathrm{M}=76 \% \text { com } \\
37,50 \pm 12,39 \text { anos. Foi utilizado o instrumento } \\
\text { ProQOL. }\end{array}$ \\
\hline $\begin{array}{l}\text { Secondary traumatic stress and burnout in } \\
\text { healthcare workers during COVID-19 outbreak } \\
\text { (Orrù et al., 2021) - } 45 \text { países diferentes. }\end{array}$ & $\begin{array}{l}\text { Avaliar o nível de burnout profissional e ETS e } \\
\text { identificar potenciais fatores de risco ou de } \\
\text { proteção entre profissionais de saúde durante o } \\
\text { surto de doença coronavírus } 2019 \text { (COVID- } \\
\text { 19). }\end{array}$ & $\begin{array}{l}\text { Transversal, } \mathrm{n}=184 \text { (médicos, enfermeiros, } \\
\text { cirurgiões, psicólogos e outros profissionais de } \\
\text { saúde), } \mathrm{H}=48,9 \% \text { e } \mathrm{M}=50,5 \% \text { com } 46,45 \pm 11,02 \\
\text { anos. Foi utilizado o instrumento STSS. }\end{array}$ \\
\hline $\begin{array}{l}\text { Sensory processing sensitivity and compassion } \\
\text { satisfaction as risk/protective factors from } \\
\text { burnout and compassion fatigue in healthcare } \\
\text { and education professionals (Pérez-Chacón et } \\
\text { al., 2021) - Países de língua espanhola } \\
\text { (México, Argentina, Colômbia, Chile, Peru, } \\
\text { República Dominicana, Costa Rica, Venezuela, } \\
\text { Guatemala, Honduras e outros) e países onde a } \\
\text { língua espanhola está fortemente implantada } \\
\text { (Estados Unidos da América e Brasil). }\end{array}$ & $\begin{array}{l}\text { Analisar a sensibilidade de processamento } \\
\text { sensorial e a satisfação por compaixão como } \\
\text { fatores de risco/proteção contra o esgotamento } \\
\text { e a FC, durante o primeiro período da } \\
\text { emergência de saúde COVID-19. }\end{array}$ & $\begin{array}{l}\text { Transversal, } \mathrm{n}=1566 \text { (profissionais da saúde e } \\
\text { da educação). Do total, } 694 \text { eram da área da } \\
\text { saúde, } \quad \mathrm{H}=4,4 \% \quad(43,58 \pm 11,88 \text { anos) e } \\
\mathrm{M}=39,91 \%(37,79 \pm 9,77 \text { anos). Foi utilizado o } \\
\text { instrumento ProQOL. }\end{array}$ \\
\hline $\begin{array}{l}\text { Social stigma during COVID-19 and its impact } \\
\text { on HCWs outcomes (Ramaci et al., 2020) - }\end{array}$ & $\begin{array}{l}\text { Identificar relações diretas e indiretas entre } \\
\text { estigma, demanda de trabalho e qualidade de }\end{array}$ & $\begin{array}{l}\text { Transversal, } n=273 \text { (médicos e enfermeiros), } \\
\mathrm{H}=49,82 \% \text { e } \mathrm{M}=50,18 \% \text { com } 46,67 \pm 8,36 \text { anos. }\end{array}$ \\
\hline
\end{tabular}




\begin{tabular}{|c|c|c|}
\hline Itália. & $\begin{array}{l}\text { vida profissional, incluindo satisfação por } \\
\text { compaixão, esgotamento e FC, em um grupo } \\
\text { de profissionais de saúde trabalhando em um } \\
\text { grande hospital no sul da Itália em uma } \\
\text { enfermaria COVID-19. }\end{array}$ & Foi utilizado o instrumento ProQOL. \\
\hline $\begin{array}{l}\text { Compassion fatigue, burnout, compassion } \\
\text { satisfaction and perceived stress in healthcare } \\
\text { professionals during the COVID-19 health } \\
\text { crisis in Spain (Ruiz-Fernández et al., 2020) - } \\
\text { Espanha. }\end{array}$ & $\begin{array}{l}\text { Avaliar a FC, o burnout, a satisfação por } \\
\text { compaixão e a percepção do estresse em } \\
\text { profissionais de saúde durante a crise de saúde } \\
\text { da doença coronavírus de } 2019 \text { (COVID-19) } \\
\text { na Espanha. }\end{array}$ & $\begin{array}{l}\text { Transversal, } n=506 \text { (médicos e enfermeiros), } \\
\mathrm{H}=23,3 \% \text { e } \mathrm{M}=76,7 \% \text { com } 46,7 \pm 10,2 \text { anos. Foi } \\
\text { utilizado o instrumento ProQOL. }\end{array}$ \\
\hline $\begin{array}{l}\text { Sintomatología asociada a trastornos de salud } \\
\text { mental en trabajadores sanitarios en Paraguay: } \\
\text { efecto COVID-19 (Samaniego et al., 2020) - } \\
\text { Paraguai. }\end{array}$ & $\begin{array}{l}\text { Determinar as prevalências de depressão, } \\
\text { ansiedade, insônia, angústia e FC, bem como } \\
\text { fatores relacionados à presença de sintomas } \\
\text { associados a esses transtornos em profissionais } \\
\text { da equipe de saúde. }\end{array}$ & $\begin{array}{l}\text { Transversal, } \mathrm{n}=126 \text { (médicos, enfermeiros, } \\
\text { psicólogos e outras profissões como } \\
\text { cinesiólogos, odontólogos, auxiliares de } \\
\text { enfermagem, entre outros), } \mathrm{H}=17,5 \% \text { e } \\
\mathrm{M}=82,5 \% \text { com } 32,22 \pm 8,233 \text { anos. Foi utilizado } \\
\text { o instrumento ProQOL. }\end{array}$ \\
\hline $\begin{array}{l}\text { The mediating role of insomnia and exhaustion } \\
\text { in the relationship between secondary traumatic } \\
\text { stress and mental health complaints among } \\
\text { frontline medical staff during the COVID-19 } \\
\text { pandemic (Secosan et al., 2020) - Romênia. }\end{array}$ & $\begin{array}{l}\text { Investigar o efeito de mediação serial de } \\
\text { insônia e exaustão na relação entre ETS e } \\
\text { queixas de saúde mental entre os profissionais } \\
\text { de saúde da linha de frente durante a pandemia } \\
\text { da COVID-19. }\end{array}$ & $\begin{array}{l}\text { Transversal, } n=126 \text { (médicos e enfermeiros), } \\
\mathrm{H}=35,7 \% \text { e } \mathrm{M}=64,3 \% \text {, não relatou dados sobre } \\
\text { a idade. Foi utilizado o instrumento STSS. }\end{array}$ \\
\hline $\begin{array}{l}\text { Psychological adjustment of healthcare workers } \\
\text { in Italy during the COVID-19 pandemic: } \\
\text { differences in stress, anxiety, depression, } \\
\text { burnout, secondary trauma, and compassion } \\
\text { satisfaction between frontline and non-frontline } \\
\text { professionals (Trumello et al., 2020) - Itália. }\end{array}$ & $\begin{array}{l}\text { Investigar o ajustamento psicológico dos } \\
\text { profissionais de saúde durante o pico da } \\
\text { pandemia da COVID-19, avaliando diferenças } \\
\text { de acordo com o trabalho ou não com } \\
\text { pacientes afetados por COVID-19 e em áreas } \\
\text { com uma propagação mais grave desta } \\
\text { pandemia. }\end{array}$ & $\begin{array}{l}\text { Transversal, } \mathrm{n}=627 \quad \text { (não relata quais } \\
\text { profissionais), } \mathrm{H}=19,9 \% \text { e } \mathrm{M}=80,1 \% \text { com } \\
40,55 \pm 11,49 \text { anos. Foi utilizado o instrumento } \\
\text { ProQOL. }\end{array}$ \\
\hline $\begin{array}{l}\text { Hardiness, stress and secondary trauma in } \\
\text { Italian healthcare and emergency workers } \\
\text { during the COVID-19 pandemic (Vagni et al., } \\
\text { 2020a) - Itália. }\end{array}$ & $\begin{array}{l}\text { Identificar e mensurar as habilidades de } \\
\text { hardiness ativadas por profissionais de saúde e } \\
\text { emergência para lidar com fatores de estresse } \\
\text { relacionados à emergência da COVID-19 que } \\
\text { podem estar associados ao risco de } \\
\text { desenvolvimento de trauma vicário ou } \\
\text { secundário. }\end{array}$ & $\begin{array}{l}\text { Transversal, } \mathrm{n}=236 \text { (apenas } 140 \text { eram do Grupo } \\
\text { Saúde: médicos, enfermeiros, psicólogos e } \\
\text { operadores). Na amostra total, eram } \mathrm{H}=41,1 \% \text { e } \\
\mathrm{M}=58,9 \% \text { com } 43,24 \pm 11,06 \text { anos. Foi utilizado } \\
\text { o instrumento STSS. }\end{array}$ \\
\hline $\begin{array}{l}\text { Coping with covid-19: emergency stress, } \\
\text { secondary trauma and self-efficacy in } \\
\text { healthcare and emergency workers in Italy } \\
\text { (Vagni et al., 2020b) - Itália. }\end{array}$ & $\begin{array}{l}\text { Identificar as estratégias de enfrentamento } \\
\text { acionadas por profissionais de saúde e } \\
\text { emergência para lidar com os fatores de } \\
\text { estresse relacionados à emergência do } \\
\text { COVID-19 que podem estar associados ao } \\
\text { risco de desenvolvimento de traumas vicários } \\
\text { ou secundários. }\end{array}$ & $\begin{array}{l}\text { Transversal, } \mathrm{n}=210 \text { (apenas } 121 \text { eram do Grupo } \\
\text { Saúde: médicos, enfermeiros, psicólogos e } \\
\text { auxiliares de saúde). Na amostra total, eram } \\
\mathrm{H}=42,9 \% \text { e } \mathrm{M}=57,1 \% \text { com } 42,13 \pm 11,35 \text { anos. } \\
\text { Foi utilizado o instrumento STSS. }\end{array}$ \\
\hline $\begin{array}{l}\text { Impact of burnout, secondary traumatic stress } \\
\text { and compassion satisfaction on hand hygiene of } \\
\text { healthcare workers during the COVID-19 }\end{array}$ & $\begin{array}{l}\text { Avaliar a prevalência de Burnout, ETS e } \\
\text { satisfação por compaixão e explorar seus } \\
\text { impactos na higiene das mãos relatada por } \\
\text { equipes médicas no período COVID-19 em }\end{array}$ & $\begin{array}{l}\text { Transversal, } \mathrm{n}=1734 \text { (médicos e enfermeiros), } \\
\mathrm{H}=24,74 \% \text { e } \mathrm{M}=75,26 \% \text { com } 33,33 \pm 6,39 \text { anos. } \\
\text { Foi utilizado o instrumento ProQOL. }\end{array}$ \\
\hline
\end{tabular}


$\mathrm{H}=$ Homens; $\mathrm{M}=$ Mulheres; ProQOL = Professional Quality of Life Scale; STSS = Secondary Traumatic Stress Scale ; ETS = Estresse Traumático Secundário; $\mathrm{FC}=$ Fadiga por Compaixão.

Fonte: Dados da pesquisa (2021).

A partir da análise dos conteúdos dos artigos, emergiram duas categorias de resultados: (1) Presença de FC nos profissionais de saúde durante a pandemia da COVID-19 e (2) Fatores relacionados à FC em profissionais de saúde durante a pandemia da COVID-19.

\section{Presença de fadiga por compaixão nos profissionais de saúde durante a pandemia da COVID-19}

Entre os nove estudos que utilizaram o ProQOL, cinco apresentaram a pontuação média na amostra total (Buselli et al., 2020; Litam \& Balkin, 2020; Pérez-Chacón et al., 2021; Ruiz-Fernández et al., 2020; Zhou et al., 2021). A maior

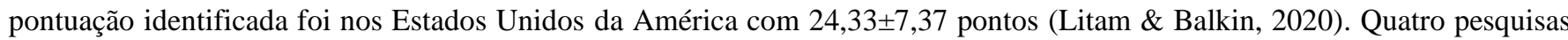
relataram as pontuações de FC de acordo com a classificação em alto, moderado e baixo (Dosil et al., 2020; Ruiz-Fernández et al., 2020; Samaniego et al., 2020; Zhou et al., 2021). Em dois estudos, os profissionais tiveram predomínio de FC média, com 19,2\% (Dosil et al., 2020) e 66,21\% (Zhou et al., 2021) e, nas duas outras investigações, os níveis foram altos, com 42,9\% (Samaniego et al., 2020) e 60,5\% (Ruiz-Fernández et al., 2020). Dois estudos não relataram a média e/ou classificação da FC na amostra total (Ramaci et al., 2020; Trumello et al., 2020).

Dos quatro estudos que adotaram o STSS para avaliação da FC, dois não apresentaram a média e/ou classificação da FC na amostra total (Vagni et al., 2020a, 2020b). Entre as outras duas outras pesquisas, o maior escore médio encontrado foi de $36,4 \pm 12,79$ com 41,3\% da amostra apresentando sintomas moderados a graves (Orrù et al., 2021).

Em seis dos 13 estudos, as mulheres apresentaram maiores pontuações de FC quando comparadas com os homens (Buselli et al., 2020; Dosil et al., 2020; Orrù et al., 2021; Ramaci et al., 2020; Ruiz-Fernández et al., 2020; Samaniego et al., 2020), sendo que em um dos estudos, o sexo feminino apresentou pontuações significativamente maiores apenas na subescala intrusão do STSS (Orrù et al., 2021). A maior pontuação identificada entre as mulheres foi na Espanha com 20,3 $\pm 7,7$ pontos (Ruiz-Fernández et al., 2020). No Paraguai, além de pontuações mais elevadas, as mulheres também apresentaram uma proporção maior de níveis altos de FC (Samaniego et al., 2020).

Em relação à idade, apenas um estudo apresentou diferenças estatisticamente significantes nas médias de FC segundo faixas etárias, onde profissionais com 56 anos ou mais tiveram pontuações mais elevadas e aqueles com 18-25 anos, valores inferiores (Dosil et al., 2020). Quanto ao número de filhos, verificou-se que profissionais de saúde sem filhos exibiram pontuações significativamente mais altas no STSS global e nos três domínios da escala (intrusão, evitação e excitação) (Orrù et al., 2021).

$\mathrm{Na}$ Itália, os enfermeiros apresentaram pontuações mais altas de FC do que médicos e/ou outros profissionais (Buselli et al., 2020). Já na Espanha, o resultado oposto foi encontrado, com maiores escores entre os médicos (Ruiz-Fernández et al., 2020). No Paraguai, tanto médicos quanto enfermeiros pontuaram mais alto quando comparados com outros profissionais de saúde da amostra (Samaniego et al., 2020). Ainda nesse mesmo estudo, os enfermeiros apresentaram uma proporção maior de FC alta $(58,6 \%)$ do que os demais profissionais (Samaniego et al., 2020).

Trabalhadores da saúde atuantes em Unidade Específica de COVID-19 para atendimento de pacientes diagnosticados apresentaram escores mais elevados de FC (Orrù et al., 2021; Ruiz-Fernández et al., 2020; Trumello et al., 2020). Os profissionais que estavam expostos à morte de pacientes apresentaram pontuações mais elevadas na escala global e nos três domínios (intrusão, evitação e excitação) do STSS (Orrù et al., 2021). Nesse mesmo estudo, aqueles que tinham familiares ou 
amigos infectados pela COVID-19 exibiram pontuações significativamente mais altas na subescala de intrusão e excitação (Orrù et al., 2021).

Dois estudos mostraram que profissionais de saúde pontuaram mais no domínio excitação do instrumento STSS, quando comparados com aqueles que atuavam na emergência (socorristas, bombeiros, integrantes da proteção civil e policiais) (Vagni et al., 2020a, 2020b). O achado demonstra que mesmo que os dois grupos prestem atendimento a pacientes com COVID-19, os profissionais da saúde parecem experimentar efeitos distintos.

Foram constatadas pontuações mais altas de FC em profissionais que acreditavam que as medidas de saúde para controle e prevenção da doença não estavam sendo respeitadas pela sociedade (Dosil et al., 2020), o que demonstra a preocupação com o contágio e as repercussões negativas associadas.

\section{Fatores relacionados à $\mathrm{FC}$ nos profissionais de saúde durante a pandemia da COVID-19}

Três dos 13 estudos elegíveis não trouxeram abordagem dos fatores relacionados devido ao objetivo estabelecido (Dosil et al., 2020; Trumello et al., 2020; Zhou et al., 2021). Ainda, dois estudos trouxeram os fatores relacionados à FC, porém, sem diferenciar quem era ou não profissional de saúde (Vagni et al., 2020a, 2020b).

Entre os fatores associados à FC destacaram-se outras condições de saúde mental como risco de depressão, transtorno geral de ansiedade (Buselli et al., 2020), estresse percebido (Ruiz-Fernández et al., 2020), esgotamento (Ruiz-Fernández et al., 2020; Secosan et al., 2020) e queixas de saúde mental mediadas por insônia e exaustão (Secosan et al., 2020).

Um estudo identificou que o estresse percebido e o domínio de despersonalização do Maslach Burnout Inventory Human Services Survey (MBI-HSS) foram relacionados à subescala de intrusão do STSS tanto entre profissionais atuantes quanto naqueles não atuantes na linha de frente da pandemia (Orrù et al., 2021). Nesse mesmo estudo, o domínio de exaustão emocional do MBI-HSS foi relacionado à subescala de intrusão do STSS somente para os trabalhadores da linha de frente (Orrù et al., 2021).

Pesquisa realizada em países de língua espanhola com profissionais da área da educação e da saúde mostrou que a facilidade de excitação e sensibilidade/consciência estética, dois domínios da sensibilidade de processamento sensorial, estavam associados à FC (Pérez-Chacón et al., 2021). Os autores afirmaram que determinadas características da sensibilidade do processamento sensorial, que fazem parte da personalidade desses profissionais, interferem na prestação do serviço e/ou cuidado e podem aumentar a suscetibilidade à FC (Pérez-Chacón et al., 2021).

A discriminação e o medo do estigma social, implicações de atuar no atendimento de pacientes com doenças altamente contagiosas como a COVID-19, também estavam relacionadas à FC (Ramaci et al., 2020). Além do contato no atendimento, a exposição dos profissionais de saúde à morte dos pacientes também contribuiu para maiores níveis de FC (Orrù et al., 2021).

Em outra vertente, as lesões morais sofridas pelos profissionais de saúde durante a pandemia também foram associadas a pontuações mais elevadas de FC, demonstrando a importância da abordagem de questões éticas (Litam \& Balkin, 2020).

Referente aos fatores sociodemográficos e ocupacional, foi verificado que o sexo feminino (Orrù et al., 2021; Samaniego et al., 2020), ser solteiro e atuar como enfermeiro (Samaniego et al., 2020) estavam relacionados ao maior risco de FC.

\section{Discussão}

A presente revisão integrativa identificou as principais características da produção científica e a presença de FC e seus fatores relacionados em profissionais de saúde durante a pandemia da COVID-19. As pesquisas identificadas foram realizadas predominantemente na Itália (Buselli et al., 2020; Dosil et al., 2020; Pérez-Chacón et al., 2021; Ramaci et al., 2020; 
Ruiz-Fernández et al., 2020; Secosan et al., 2020; Trumello et al., 2020; Vagni et al., 2020a, 2020b) um dos países que enfrentou a epidemia inicial da doença, com picos de casos em março e abril de 2020 (Committee for the Coordination of Statistical Activities, 2020).

O instrumento mais utilizado para avaliar a FC nos estudos elegíveis foi o ProQOL. Trata-se de um instrumento frequentemente adotado nos estudos e que pode ser utilizado com êxito na mensuração da prevalência e predição do risco de FC (Cavanagh et al., 2020). Em relação ao delineamento dos estudos identificados, todos eram transversais, o que indica a necessidade de explorar abordagens metodológicas que permitam o estabelecimento da relação de causalidade entre as variáveis investigadas, e de propor ensaios clínicos randomizados com objetivo de minimizar e/ou prevenir a FC nesse contexto.

O presente estudo mostrou que profissionais de saúde no período da pandemia apresentavam FC. Antes do surgimento do novo coronavírus, esse público já manifestava essa condição, como foi constatado em revisão sistemática com metanálise de 71 estudos realizados até dezembro de 2017 (Cavanagh et al., 2020). O achado representa um alerta para o tratamento dos casos, visto que a FC traz repercussões não somente para o profissional, mas também no cuidado prestado, uma vez que favorece atitudes como iatrogenias, distanciamento e negação do outro, além da esquiva de responsabilidades e tomada de decisões (Souza et al., 2019).

De forma geral, tanto médicos quanto enfermeiros predominaram nas amostras e foram os mais afetados pela FC nos estudos elegíveis. Estudo realizado em um serviço de urgência e emergência de um hospital universitário de Portugal, mostrou que enfermeiros tinham níveis elevados de FC (Borges et al., 2019). Essa categoria profissional tem maior risco de desenvolver problemas de ordem emocional, devido ao contato direto e maior envolvimento afetivo com pacientes e familiares (Dosil et al., 2020). Por outro lado, médicos também estão sujeitos às situações críticas como a responsabilidade de informar a condição clínica dos doentes, além do enfrentamento de consequências resultantes da tomada de decisões difíceis, das quais se destacam as lesões morais que estão relacionadas à FC (Litam \& Balkin, 2020).

Além do fardo da rotina, precarização das condições no ambiente de trabalho e contato com aspectos emocionais tanto dos pacientes quanto dos familiares (Teixeira et al., 2020), os profissionais de saúde podem apresentar maior sensibilidade de processamento sensorial que interferem nas relações com a pessoa cuidada, tornando-os mais vulneráveis à FC devido às respostas exacerbadas aos estímulos (Pérez-Chacón et al., 2021).

Outro achado identificado neste estudo foi o predomínio do sexo feminino nos artigos elegíveis, que reflete a representatividade desse grupo a nível mundial no setor saúde (Committee for the Coordination of Statistical Activities, 2020). A maior vulnerabilidade ao risco de contaminação pelo vírus (Teixeira et al., 2020) e o estresse emocional ocasionado por situações vivenciadas por mulheres, como o contato constante com pacientes em Unidades de Terapia Intensiva, alocação em atividades menos qualificadas, salários baixos, discriminação, situações de violência e demandas de cuidado fora do ambiente de trabalho (Committee for the Coordination of Statistical Activities, 2020), podem, possivelmente, explicar as maiores pontuações e risco de FC nesse grupo.

Apenas um estudo identificado na revisão mostrou que profissionais com idade superior a 56 anos apresentaram maiores pontuações de FC (Dosil et al., 2020). O achado diverge de estudo realizado em Portugal, onde enfermeiros mais jovens expressaram níveis mais altos dessa condição (Borges et al., 2019). Sugere-se a realização de mais estudos que possam investigar duas possíveis hipóteses entre idade e anos de experiência, na qual profissionais mais jovens podem ser mais suscetíveis à FC por estarem se habituando as rotinas e iniciando o contato com os casos, e aqueles com idade mais avançada por sentirem a sobrecarga dos anos de trabalho e por assumirem mais responsabilidades.

De forma semelhante, somente um estudo elegível mostrou que profissionais que não tinham filhos apresentaram maiores pontuações no STSS (Orrù et al., 2021) e outro, constatou que ser solteiro estava relacionado com maiores pontuações 
de FC (Samaniego et al., 2020). Este último achado se assemelha ao resultado de pesquisa realizada com enfermeiros na Turquia (Aslan et al., 2021). Embora as repercussões negativas da FC (Stamm, 2010) e o medo de contaminar os familiares existam por parte dos profissionais (Teixeira et al., 2020), sugere-se a hipótese de que ter parceiro ou filhos podem auxiliar no enfrentamento dessa condição.

Em relação as questões diretamente relacionadas à pandemia, verificou-se no presente estudo que profissionais que atendiam em Unidades Específicas para COVID-19 (Orrù et al., 2021; Ruiz-Fernández et al., 2020; Trumello et al., 2020) que presenciavam a morte de pacientes e tinham amigos ou familiares infectados pelo vírus apresentaram maiores pontuações de FC (Orrù et al., 2021). Profissionais da linha de frente da COVID-19 experimentam situações que podem elucidar a presença da FC, como a constante preocupação e envolvimento com os pacientes, distúrbios do sono, irritabilidade e evitação de tarefas que remetam ao trauma dos doentes (Litam \& Balkin, 2020). Acrescenta-se a esse contexto, o medo de novos surtos de casos e os transtornos psicológicos nos profissionais que acreditam que as medidas preventivas não estão sendo respeitadas pela sociedade (Dosil et al., 2020).

Devido ao alto potencial de propagação do novo coronavírus (World Health Organization, 2020), profissionais de saúde que atendem os casos da doença também têm enfrentado os efeitos do estigma social, como os prejuízos no convívio social e na saúde mental (Borges et al., 2021). Esse processo está relacionado à FC e caracteriza-se por sentimentos de medo e atitudes discriminatórias (Ramaci et al., 2020), desafiando o profissional a lidar com sentimentos contraditórios de antipatia (Souza et al., 2019) e empatia relacionada ao ato de cuidar (Stamm, 2010).

As condições de saúde mental associadas à FC identificadas na presente revisão são bastante frequentes nos profissionais de saúde de forma geral, o que está de acordo com estudo de revisão sistemática que identificou depressão, ansiedade e estresse como as mais prevalentes (Vizheh et al., 2020). Semelhante à FC, o esgotamento também está associado ao risco de depressão e transtorno geral de ansiedade (Buselli et al., 2020) e em alguns estudos com a própria FC (Ruiz-Fernández et al., 2020), o que está coerente com a literatura, visto que se trata de um componente da FC (Stamm, 2010). Este trabalho evidencia a presença de FC em profissionais de saúde na pandemia, o que demonstra que esse período tem intensificado os prejuízos mentais oriundos da exposição ao trauma e sofrimento do outro. Os achados alertam para a necessidade de ampliação do olhar para os custos do cuidado e de desenvolvimento de estratégias e intervenções, com objetivo de contribuir para a redução do sofrimento mental entre os profissionais de saúde.

Como limitação desta revisão integrativa, aponta-se a impossibilidade de estimar a prevalência da FC, pois os dados dos estudos variaram na forma de apresentação, seja por meio de médias, classificação ou até mesmo ausência da informação. Deve-se considerar também que a utilização de diferentes instrumentos para avaliação da FC não permitiu a padronização na avaliação e comparação entre os estudos.

\section{Conclusão}

Em resposta à questão de pesquisa, foi identificado que a produção científica sobre a FC em profissionais de saúde durante a pandemia da COVID-19 se concentra em estudos de delineamento transversal, realizados em países europeus como a Itália, com predomínio de mulheres, médicos e enfermeiros nas amostras. O principal instrumento utilizado para investigação da FC foi o ProQOL.

Nesse contexto, verificou-se que os profissionais de saúde apresentam algum nível de FC, de forma mais acentuada em alguns grupos como as mulheres, médicos, enfermeiros e aqueles atuantes em unidades específicas de atendimento a pacientes com COVID-19. Além disso, foram identificadas questões de saúde mental, éticas, ocupacionais e sociodemográficas como fatores relacionados aos níveis mais elevados de FC.

Os achados desta revisão fornecem um alerta para que as instituições, gestores e equipes de saúde busquem a 
capacitação sobre os riscos de sofrimentos psicológicos no trabalho, sobretudo a FC, e proporcionem suporte aos profissionais de saúde a partir de educação em saúde, monitoramento regular para rastreio, abordagens específicas de tratamento e acompanhamento dos casos de FC, considerando os fatores de risco e grupos mais suscetíveis, para que estes sejam priorizados.

A condução de novos estudos a partir dos resultados dessa revisão integrativa também é necessária, principalmente ensaios clínicos randomizados, com objetivo de testar intervenções que possam auxiliar os profissionais de saúde a reduzir os efeitos negativos da FC e, consequentemente, melhorar a qualidade da atenção e cuidado voltados para esse público.

\section{Agradecimentos}

O presente trabalho foi realizado com apoio da Coordenação de Aperfeiçoamento de Pessoal de Nível Superior Brasil (CAPES) - Código de Financiamento 001.

\section{Referências}

Aslan, H., Erci, B., \& Pekince, H. (2021). Relationship between compassion fatigue in nurses, and work-related stress and the meaning of Life. Journal of Religion and Health, 2, 1-13. https://doi.org/10.1007/s10943-020-01142-0

Borges, E. M. das N., Fonseca, C. I. N. da S., Baptista, P. C. P., Queirós, C. M. L., Baldonedo-Mosteiro, M., \& Mosteiro-Diaz, M. P. (2019). Compassion fatigue among nurses working on an adult emergency and urgent care unit. Revista Latino-Americana de Enfermagem, 27. https://doi.org/10.1590/15188345.2973.3175

Borges, T. P., Schulz, R. da S., Magalhães, J. B. de, Campos, L. M., Anjos, K. F. dos, \& Rosa, D. de O. S. (2021). Stigmas related to Covid-19 and their prevention. Physis: Revista de Saúde Coletiva, 31(1), 1-6. https://doi.org/10.1590/s0103-73312021310103

Buselli, R., Corsi, M., Baldanzi, S., Chiumiento, M., Del Lupo, E., Dell’Oste, V., Bertelloni, C. A., Massimetti, G., Dell'Osso, L., Cristaudo, A., \& Carmassi, C. (2020). Professional quality of life and mental health outcomes among health care workers exposed to Sars-Cov-2 (Covid-19). International Journal of Environmental Research and Public Health, 17(17). https://dx.doi.org/10.3390/ijerph17176180

Cavanagh, N., Cockett, G., Heinrich, C., Doig, L., Fiest, K., Guichon, J. R., Page, S., Mitchell, I., \& Doig, C. J. (2020). Compassion fatigue in healthcare providers: A systematic review and meta-analysis. Nursing Ethics, 27(3), 639-665. https://doi.org/10.1177/0969733019889400

Committee for the Coordination of Statistical Activities. (2020). How COVID-19 is changing the world: A statistical perspective, Volume II. UNICEF DATA. https://unstats.un.org/unsd/ccsa/documents/covid19-report-ccsa_vol2.pdf

Dosil, M., Ozamiz-Etxebarria, N., Redondo, I., Picaza, M., \& Jaureguizar, J. (2020). Psychological symptoms in health professionals in Spain after the first wave of the COVID-19 pandemic. Frontiers in Psychology, 11. https://doi.org/10.3389/fpsyg.2020.606121

Figley, C. R. (2002). Compassion fatigue: Psychotherapists' chronic lack of self-care. Journal of Clinical Psychology, 58(11), 1433-1441. https://doi.org/10.1002/jclp. 10090

Litam, S. D. A., \& Balkin, R. S. (2020). Moral injury in health-care workers during COVID-19 pandemic. Traumatology, 27(1), 14-19. https://doi.org/10.1037/trm0000290

McCann, I. L., \& Pearlman, L. A. (1990). Vicarious traumatization: A framework for understanding the psychological effects of working with victims. Journal of Traumatic Stress, 3(1), 131-149. https://doi.org/10.1002/jts.2490030110

Mendes, K. D. S., Silveira, R. C. de C. P., \& Galvão, C. M. (2008). Revisão integrativa: Método de pesquisa para a incorporação de evidências na saúde e na enfermagem. Texto \& Contexto - Enfermagem, 17(4), 758-764. https://doi.org/10.1590/S0104-07072008000400018

Orrù, G., Marzetti, F., Conversano, C., Vagheggini, G., Miccoli, M., Ciacchini, R., Panait, E., \& Gemignani, A. (2021). Secondary traumatic stress and Burnout in healthcare workers during COVID-19 outbreak. International Journal of Environmental Research and Public Health, 18(1). https://dx.doi.org/10.3390/ijerph18010337

Page, M. J., McKenzie, J. E., Bossuyt, P. M., Boutron, I., Hoffmann, T. C., Mulrow, C. D., Shamseer, L., Tetzlaff, J. M., Akl, E. A., Brennan, S. E., Chou, R., Glanville, J., Grimshaw, J. M., Hróbjartsson, A., Lalu, M. M., Li, T., Loder, E. W., Mayo-Wilson, E., McDonald, S., ... Moher, D. (2021). The PRISMA 2020 statement: An updated guideline for reporting systematic reviews. BMJ, 372, n71. https://doi.org/10.1136/bmj.n71

Pérez-Chacón, M., Chacón, A., Borda-Mas, M., \& Avargues-Navarro, M. L. (2021). Sensory processing sensitivity and compassion satisfaction as risk/protective cactors from Burnout and compassion fatigue in healthcare and education professionals. International Journal of Environmental Research and Public Health, 18(2). https://dx.doi.org/10.3390/ijerph18020611

Ramaci, T., Barattucci, M., Ledda, C., \& Rapisarda, V. (2020). Social stigma during COVID-19 and its impact on HCWs outcomes. Sustainability, 12(9), 3834. https://doi.org/10.3390/su12093834 
Ruiz-Fernández, M. D., Ramos-Pichardo, J. D., Ibáñez-Masero, O., Cabrera-Troya, J., Carmona-Rega, M. I., \& Ortega-Galán, Á. M. (2020). Compassion fatigue, burnout, compassion satisfaction and perceived stress in healthcare professionals during the COVID-19 health crisis in Spain. Journal of Clinical Nursing, 29(21-22), 4321-4330. https://doi.org/10.1111/jocn.15469

Samaniego, A., Urzúa, A., Buenahora, M., \& Vera-Villarroel, P. (2020). Sintomatología asociada a trastornos de salud mental en trabajadores sanitarios en paraguay: Efecto COVID-19. [Symptomatology associated with mental health disorders in health workers in Paraguay: COVID-19 effect.]. Revista Interamericana de Psicología, 54(1), 1-19. https://doi.org/10.30849/ripijp.v54i1.1298

Secosan, I., Virga, D., Crainiceanu, Z. P., \& Bratu, T. (2020). The mediating role of insomnia and exhaustion in the relationship between secondary traumatic stress and mental health complaints among frontline medical staff during the COVID-19 pandemic. Behavioral Sciences, 10(11), 164. https://dx.doi.org/10.3390/bs10110164

Souza, C. G. V. M. de, Benute, G. R. G., Moretto, M. L. T., Levin, A. S. S., Assis, G. R. de, Padoveze, M. C., \& Lobo, R. D. (2019). Qualidade de vida profissional na saúde: Um estudo em Unidades de Terapia Intensiva. Estudos de Psicologia (Natal), 24(3), 269-280. https://doi.org/10.22491/16784669.20190028

Stamm, B. H. (2010). The Concise ProQOL Manual. https://proqol.org/uploads/ProQOLManual.pdf

Stetler, C. B., Morsi, D., Rucki, S., Broughton, S., Corrigan, B., Fitzgerald, J., Giuliano, K., Havener, P., \& Sheridan, E. A. (1998). Utilization-focused integrative reviews in a nursing service. Applied Nursing Research: ANR, 11(4), 195-206. https://doi.org/10.1016/s0897-1897(98)80329-7

Teixeira, C. F. de S., Soares, C. M., Souza, E. A., Lisboa, E. S., Pinto, I. C. de M., Andrade, L. R. de, \& Espiridião, M. A. (2020). A saúde dos profissionais de saúde no enfrentamento da pandemia de Covid-19. Ciência \& Saúde Coletiva, 25, 3465-3474. https://doi.org/10.1590/1413-81232020259.19562020

Trumello, C., Bramanti, S. M., Ballarotto, G., Candelori, C., Cerniglia, L., Cimino, S., Crudele, M., Lombardi, L., Pignataro, S., Viceconti, M. L., \& Babore, A. (2020). Psychological adjustment of healthcare workers in Italy during the COVID-19 pandemic: Differences in stress, anxiety, depression, Burnout, secondary trauma, and compassion satisfaction between frontline and non-frontline professionals. International Journal of Environmental Research and Public Health, 17(22). https://dx.doi.org/10.3390/ijerph17228358

Ursi, E. S. (2005). Prevenção de lesões de pele no perioperatório: Revisão integrativa da literatura [Dissertação, Universidade de São Paulo]. https://doi.org/10.11606/D.22.2005.tde-18072005-095456

Vagni, M., Maiorano, T., Giostra, V., \& Pajardi, D. (2020a). Hardiness, stress and secondary trauma in italian healthcare and emergency workers during the COVID-19 pandemic. Sustainability, 12(14), 5592. https://doi.org/10.3390/su12145592

Vagni, M., Maiorano, T., Giostra, V., \& Pajardi, D. (2020b). Coping with COVID-19: Emergency stress, secondary trauma and self-efficacy in healthcare and emergency workers in Italy. Frontiers in Psychology, 11, 566912-566912. https://doi.org/10.3390/su12145592

Vizheh, M., Qorbani, M., Arzaghi, S. M., Muhidin, S., Javanmard, Z., \& Esmaeili, M. (2020). The mental health of healthcare workers in the COVID-19 pandemic: A systematic review. Journal of Diabetes and Metabolic Disorders, 19(2), 1967-1978. https://doi.org/10.1007/s40200-020-00643-9

World Health Organization. (2020). Report of the WHO-China Joint Mission on Coronavirus Disease 2019 (COVID-19). https://www.who.int/docs/defaultsource/coronaviruse/who-china-joint-mission-on-covid-19-final-report.pdf

World Health Organization. (2021). WHO Coronavirus (COVID-19) Dashboard. https://covid19.who.int

Zhou, Q., Lai, X., Wan, Z., Zhang, X., \& Tan, L. (2021). Impact of burnout, secondary traumatic stress and compassion satisfaction on hand hygiene of healthcare workers during the COVID-19 pandemic. Nursing Open, 00, 1-7. https://doi.org/10.1002/nop2.786 